\title{
PRESSÃO ARTERIAL E ATENÇÃO FARMACÊUTICA: O CUIDADO FAZ A DIFERENÇA
}

\author{
BLOOD PRESSURE AND PHARMACEUTICAL CARE: CARE MAKES THE DIFFERENCE
}

\author{
Lília Gabriela Andrade Silva ${ }^{\mathrm{a}^{*}}$, Cicera Cristina Vidal Aragão ${ }^{\mathrm{a}^{*}}$, Wilson Sabino ${ }^{\mathbf{b}^{* * *}}$ \\ acristina.vidal@uscs.edu.br, bwilsonsabino14@gmail.com \\ *Universidade Municipal de São Caetano do Sul - São Caetano do Sul (SP), Brasil \\ **Universidade Federal do Oeste do Pará - Santarém (PA), Brasil
}

Data de recebimento do artigo: 12/06/2015

Data de aceite do artigo: 17/09/2015

\section{RESUMO}

Introdução: A hipertensão arterial sistêmica (HAS) é reconhecida como principal fator de risco para doença cerebrovascular, doença arterial coronariana, insuficiência renal crônica e doença vascular de extremidades. Quando náo tratada adequadamente, a HAS pode resultar em graves consequências a órgãos vitais como coração, cérebro e rins, constituindo, desse modo, um dos mais graves problemas de saúde pública. Nesse sentido, o emprego de terapêutica medicamentosa, além de ações educativas para mudanças no estilo de vida, é considerado elemento necessário para atingir o seu controle. Objetivos: Verificar a mudança de valores de pressão arterial entre os usuários que recebem o cuidado da atenção farmacêutica. Metodologia: O estudo prospectivo, controlado, foi desenvolvido na Farmácia-Escola da Universidade Municipal de São Caetano do Sul (USCS). Para avaliar o efeito da prática da atençáo farmacêutica sobre o controle da pressão arterial em usuários hipertensos, utilizou-se o método Dáder. Resultados: A média de idade dos 18 usuários de medicamentos para hipertensão arterial foi de $69,7 \pm 8,9$ anos. Foram prescritos a esses usuários 120 princípios ativos (média de $6,7 \pm 3,8$ medicamentos por prescriçáo). Ao final do acompanhamento a redução média da pressão arterial sistólica foi de $23,8 \mathrm{mmHg}(\mathrm{p}<0,002)$. Conclusão: A atenção farmacêutica, realizada de maneira sistemática pelo farmacêutico treinado para esta finalidade, pode contribuir efetivamente para o controle da pressão arterial dos usuários de medicamentos.

Palavras-chave: Hipertensão; atenção farmacêutica; farmacoterapia.

\section{ABSTRACT}

Introduction: Systemic Arterial Hypertension (SAH) is known as the main risk factor for cerebrovascular disease, coronary artery disease, chronic kidney failure, and peripheral vascular disease. If not properly treated, SAH can result in severe consequences to vital organs such as heart, brain, and kidneys, thus constituting one of the most severe problems of public health. Accordingly, the use of medication therapy and educational campaigns for changes in lifestyle are considered a necessary aspect for the SAH control. Object: This study aims to determine the change in blood pressure values among users receiving pharmaceutical care. Methods: A prospective, controlled study to evaluate the effect of the practice of pharmaceutical care on blood pressure control in hypertensive patients, conducted at the Teaching Pharmacy of the Municipal University of São Caetano do Sul (USCS). The Dáder method was used. Results: For 18 arterial hypertension drug users, the average age was $69.7 \pm 8.9$ years. One hundred and twenty active ingredients were prescribed to these users, with an average of $6.7 \pm 3.8$ drugs per prescription. The systolic blood pressures of the studied group at the end of follow-up showed an average reduction of $23.8 \mathrm{mmHg}$ $(\mathrm{p}<0.002)$. Conclusion: The pharmaceutical care, carried out systematically by pharmacists trained for this purpose, can effectively contribute to the control of blood pressure of medication users.

Keywords: Hypertension; pharmaceutical care; pharmacotherapy. 


\section{Introdução}

Nos hospitais ao redor do mundo, em torno de 15 a $20 \%$ dos orçamentos sáo gastos com tratamentos decorrentes aos agravos relacionados ao mau uso dos medicamentos ${ }^{1}$. Nos Estados Unidos, 7,9 a 17\% das hospitalizaçóes têm como responsáveis as reações adversas provocadas pelos medicamentos ${ }^{2}$. Já no Brasil, os medicamentos ocupam o primeiro lugar entre os agentes causadores de intoxicaçôes em seres humanos e o segundo lugar nos registros de mortes por intoxicação ${ }^{1}$.

Na visão de Cipolle, Strand e Morley ${ }^{3}$, não deveríamos nos preocupar somente com o custo dos produtos farmacêuticos, mas também com o custo do emprego inadequado dos medicamentos. Segundo esses pesquisadores, as tentativas para reduzir as despesas com medicamentos vêm apresentando resultado limitado por não ter, até então, enfoque voltado à atenção específica dos pacientes.

Contudo, até o final da década de 1980, o farmacêutico náo contava com um procedimento centrado no cuidado do paciente fora da área hospitalar, com o objetivo de minimizar os agravos de saúde decorrentes do medicamento. Em 1990, voltou-se o olhar, de maneira mais sistematizada, para a relação entre paciente e farmacêutico no âmbito comunitário, definindo-se, assim, a atenção farmacêutica, como "aquela em que o profissional farmacêutico toma para si a responsabilidade da terapia medicamentosa com a finalidade de alcançar resultados definitivos que melhorem a qualidade de vida dos pacientes" ${ }^{\prime}$.

Após esse momento, percebeu-se que já não bastava mais uma atenção voltada somente ao tecnicismo da droga, ao mesmo tempo faz-se necessária uma prática direcionada à necessidade do paciente, centrada na identificação, prevenção e resolução de problemas relacionados aos medicamentos ${ }^{5,6}$.

Entretanto, para entender as necessidades de cada usuário, faz-se necessário que o farmacêutico possua conhecimentos também em ciências humanas e sociais, incluindo a área pedagógica, para uma boa atuação direcionada às necessidades do paciente ${ }^{7}$. Não é suficiente o acesso do paciente aos serviços de saúde e aos próprios medicamentos para garantir o êxito de um tratamento. A este acesso deve ser acrescentada a prática da Atenção Farmacêutica $^{8}$, com metodologia apropriada, pois ela influencia positivamente a terapêutica, especialmente nos portadores de doenças crônicas.

As enfermidades cardiovasculares, como parte desse conjunto de doenças crônicas, são as principais causas de morte em populaçóes com taxas baixas de mortalidade, pois os riscos da morbimortalidade por elas estão relacionados com o aumento da idade.

No Brasil, em 2010, as doenças circulatórias representavam $28 \%$ das causas de falecimento, sendo as primeiras causas de óbito, com taxa de 148,8 por 100 mil habitantes?. Todavia, apesar de alguns estudos demonstrarem a diminuição desses falecimentos ${ }^{10,11}$, possivelmente motivada pela melhora da qualidade de vida $^{12}$, eles podem se apresentar desigualmente nos grupos populacionais de um mesmo país ${ }^{13}$. Possivelmente parte desse descenso seja motivado pela detecçáa precoce desse tipo de enfermidade, seguido de políticas e programas que proporcionam maior acesso da populaçáo aos medicamentos considerados essenciais. Entretanto, como observado em diversos estudos ${ }^{7,14,15}$, o maior acesso aos programas e aos medicamentos não garante, por si só, um melhor controle dos fatores de risco, a exemplo da hipertensão arterial.

Com objetivo de avaliar a assistência a pessoas com hipertensão arterial e sua adesão ao tratamento, autores observaram que, apesar de, no acesso e consulta, essas pessoas se mostrarem satisfeitas com o atendimento, havia baixa proporção de pessoas com pressão arterial controla$\mathrm{da}^{16}$. Contudo, pesquisas demonstram que a baixa adesão ao tratamento anti-hipertensivo se relaciona com a baixa qualidade de informaçáo do usuário no que diz respeito a seu tratamento, somada aos efeitos colaterais produzidos pelo processo medicamentoso ${ }^{17,18,19}$. Não obstante, esses fatos apontam náo somente para necessidade de detecção precoce deste fator de risco, mas, principalmente, para a necessidade do acompanhamento farmacoterapêutico por profissional qualificado com objetivo de melhorar a qualidade de vida do paciente hipertenso.

Estudos vêm demonstrando essa imprescindível necessidade, afirmando que serviços clínicos realizados por farmacêuticos vinculados ao paradigma da atenção farmacêutica podem contribuir para melhor adesão medicamentosa, com subsequente diminuição da pressão arterial dos pacientes não controlados ${ }^{14,20}$. Por essa razão, este estudo se propóe a verificar a mudança de valores de pressão arterial entre os usuários que recebem o cuidado da atenção farmacêutica em uma farmácia-escola.

\section{Metodologia}

Trata-se de estudo do tipo prospectivo e controlado, em que os indivíduos hipertensos foram selecionados através de uma amostragem por conveniência na Farmácia-Escola da Universidade Municipal de São Caetano do Sul (FarmaUSCS). Este local foi inaugurado em 2003, estruturando-se a partir de uma parceria com a Secretaria de Saúde de São Caetano do Sul, integrando-se ao Programa de Assistência Farmacêutica Municipal. Os medicamentos manipulados nesse estabelecimento são distribuídos aos usuários sem custos, atendendo a prescriçóes médicas provenientes da rede pública e privada do município. 
Considerou-se o conceito de hipertensão arterial sistêmica (HAS) descrito nas VI Diretrizes Brasileiras de Hipertensão ${ }^{21}$ que define hipertensão como níveis de pressão arterial sistólica (PAS) $\geq 140 \mathrm{mmHg}$ e/ou de pressão arterial diastólica (PAD) $\geq 90 \mathrm{mmHg}$. Foi considerado hipertenso controlado o indivíduo com diagnóstico prévio de HAS, que apresentou uma média aritmética de duas ou três medidas de pressão arterial em membro superior do paciente, na posição sentada, inferiores a $140 \mathrm{mmHg}$ para a PAS e $90 \mathrm{mmHg}$ para a $\mathrm{PAD}$. No caso do hipertenso não controlado considerou-se o indivíduo que apresentou a média dos níveis pressóricos $\geq 140 \mathrm{mmHg}$ para a PAS e $\geq 90 \mathrm{mmHg}$ para a PAD. A pressão arterial foi obtida por pesquisador treinado para este fim, realizando-se três mediçôes e obtendo-se a média das duas últimas.

Os critérios de inclusão foram: ser portador de HAS; ter idade igual ou superior a 18 anos; aceitar participar do estudo, por meio de consentimento livre e esclarecido. Critérios de exclusão: mulher portadora de doença hipertensiva específica de gestação, usuários portadores de doenças crônicas degenerativas debilitantes, limitada autonomia e poder de decisão, e pacientes com hipertensão secundária.

Verificou-se a legibilidade das prescriçóes medicamentosas, classificando as prescriçōes em legível, pouco legível e ilegível ${ }^{22}$. As prescriçóes foram avaliadas por alunos do primeiro ano de farmácia, que não apresentavam ainda experiência em farmácias e drogarias, com objetivo único de isentar-se dos vícios de leitura.

Na observância do perfil farmacoterapêutico, os medicamentos apresentados pela nomenclatura constante na Denominação Comum Brasileira (DCB) foram classificados pelo Índice de Classificação Anatômico Químico e Terapêutico (ATC), para o conhecimento das classes terapêuticas mais prescritas.

Para o acompanhamento da história farmacoterapêutica do paciente e a correta identificaçáo dos Resultados Clínicos Negativos associado ao uso do Medicamento (RNM) utilizou-se o método Dáder ${ }^{23}$, que, segundo o Consenso de Granada, estabelece uma classificação de RNM em seis categorias, que, por sua vez, se agrupam em três subcategorias: "necessidade", "efetividade" e "segurança".

Durante a intervençáo foi adotado como procedimento o acompanhamento farmacoterapêutico (atenção farmacêutica). A entrevista inicial ocorreu no momento em que se firmou o consentimento informado; outras entrevistas foram pré-agendadas, em um período de quatro meses. Foi realizada a avaliação dos possíveis PRM e proposto um plano de atenção farmacêutica. Para tal procedimento, na primeira entrevista, utilizou-se de formulários de seguimento terapêutico.
Como parte do processo de investigação foi criado um banco de dados no programa de planilhas da Microsoft (Excel), posteriormente codificadas, e transferidas para o programa estatístico Statistical Package For The Social Sciences (SPSS) for Windows para as devidas análises. Para a comparaçáo das variáveis contínuas utilizou-se do teste $t$ de Student, considerando o nível de significância $\mathrm{p}<0,05$.

Este projeto conta com aprovação do Comitê de Ética em Pesquisa da Universidade Municipal de Sáo Caetano do Sul no 247.032.

\section{Resultados}

Dos 18 usuários de medicamentos para hipertensão atendidos na Farmácia-Escola da USCS, a média de idade encontrada foi de $69,7 \pm 8,9$ anos, sendo $71 \pm 9,5$ anos para o sexo masculino $(33,3 \%)$ e $69 \pm 9,1$ anos para o sexo feminino $(66,7 \%)$, não sendo encontrada diferença estatisticamente significativa entre essas médias.

Foram prescritos a esses usuários 120 princípios ativos, apresentando a média de $6,7 \pm 3,8$ medicamentos por prescrição, sendo $7,5 \pm 4,1$ medicamentos por receita para as pessoas do sexo feminino e $5 \pm 2,5$ medicamentos por receita para o sexo masculino, náo sendo encontrada diferença estatisticamente significante na quantidade de medicamentos prescritos entre os sexos.

Observa-se, na Tabela 1 , que $61,1 \%$ das prescrições contêm mais de quatro princípios ativos, denotando que $44,4 \%$ das receitas que apresentam essa quantidade de medicamentos variam quanto a sua legibilidade, ou seja, de pouco legível $(33,3 \%)$ a ilegível.

Tabela 1: Frequência quanto à quantidade de medicamentos por prescrição e a grafia encontrada nas prescrições de medicamentos apresentadas na Farmácia e Fisioterapia da USCS. Município de São Caetano do Sul, SP, 2013.

\begin{tabular}{|c|c|c|c|c|c|c|c|}
\hline \multirow{2}{*}{$\begin{array}{l}\text { Quantidade } \\
\text { de medica- } \\
\text { mento por } \\
\text { prescriçáa }\end{array}$} & \multicolumn{6}{|c|}{ Legibilidade } & \multirow[b]{2}{*}{ Total } \\
\hline & & Legível & & $\begin{array}{l}\text { Pouco } \\
\text { egível }\end{array}$ & Ilegível & & \\
\hline 1 a 4 & 2 & $(11,1 \%)$ & 3 & $(16,7 \%)$ & $2(11,1 \%)$ & 7 & $(38,9 \%)$ \\
\hline 5 a 9 & 3 & $(16,7 \%)$ & 4 & $(22,2 \%)$ & $-\quad-$ & 7 & $(38,9 \%)$ \\
\hline 10 a 17 & - & - & 2 & $(11,1 \%)$ & $2(11,1 \%)$ & 4 & $(22,2 \%)$ \\
\hline Total & 5 & $(27,8 \%)$ & 9 & $(50,0 \%)$ & $4 \quad(22,2 \%)$ & 18 & (100\%) \\
\hline
\end{tabular}

Fonte: Adaptado da Farmácia-Escola da Universidade Municipal de Sáo Caetano do Sul.

$\mathrm{Na}$ Tabela 2 são apresentados os grupos de medicamentos mais prescritos aos usurários atendidos na farmácia-escola e clínica de fisioterapia da USCS, de acordo com a classificação anatômica (Nível 1) e terapêutica (Nível 2) da ATC. Em relação à primeira, 56,6\% dos 
medicamentos receitados atuam sobre o sistema cardiovascular, seguido por trato alimentar e metabolismo $(23,2 \%)$, aparelho locomotor $(8,1 \%)$ e medicamentos com ação sobre o sistema nervoso (5\%). Considerando a classificação anátomo-terapêutica, entre os medicamentos mais consumidos figuram cinco com ação sobre o sistema cardiovascular: agentes inibidores do sistema renina-angiotensina $(18,2 \%)$, antilipidêmicos $(14,1 \%)$, diuréticos $(12,1 \%)$, betabloqueadores e bloqueadores dos canais de cálcio (10,1\% somados). Seguem-se a estes os medicamentos utilizados na diabetes $(8,1 \%)$, antiácidos/antiulcerosos/antiflatulentos $(7,1 \%)$ e vitaminas e suplementos minerais (6\%).

Tabela 2: Distribuição dos medicamentos utilizados pelos usuários da Farmácia e Fisioterapia da USCS, segundo classificação anátomo-terapêutica (níveis 1 e 2 da Anatomical Therapeutical Chemical Index - ATC). Município de São Caetano do Sul, SP, 2013.

\begin{tabular}{|c|c|c|c|}
\hline Medicamentos & $\begin{array}{l}\text { Código } \\
\text { ATC }\end{array}$ & $\begin{array}{l}\mathrm{N}^{\circ} \\
\mathrm{Abs}\end{array}$ & $\%$ \\
\hline Sistema cardiovascular & C & 56 & 56,6 \\
\hline $\begin{array}{l}\text { Agentes sobre o sistema } \\
\text { renina-angiotensina }\end{array}$ & $\mathrm{C} 09$ & 18 & 18,2 \\
\hline Antilipêmicos & $\mathrm{C} 10$ & 14 & 14,1 \\
\hline Diuréticos & $\mathrm{C} 03$ & 12 & 12,1 \\
\hline Betabloqueadores & $\mathrm{C} 07$ & 06 & 6,1 \\
\hline $\begin{array}{l}\text { Bloqueadores dos canais de } \\
\text { cálcio }\end{array}$ & $\mathrm{C} 08$ & 04 & 4,0 \\
\hline Cardioterápicos & C01 & 02 & 2,0 \\
\hline $\begin{array}{l}\text { Trato alimentar e } \\
\text { metabolismo }\end{array}$ & A & 23 & 23,2 \\
\hline $\begin{array}{l}\text { Medicamentos usados no } \\
\text { diabetes }\end{array}$ & A10 & 8 & 8,1 \\
\hline $\begin{array}{l}\text { Antiácidos/Antiulcerosos/ } \\
\text { Antiflatulentos }\end{array}$ & A02 & 7 & 7,1 \\
\hline Vitaminas & A11 & 4 & 4,0 \\
\hline Suplementos minerais & A12 & 2 & 2,0 \\
\hline Distúrbios gastrintestinais & A03 & 1 & 1,0 \\
\hline Preparaçôes antiobesidade & A08 & 1 & 1,0 \\
\hline Aparelho locomotor & $\mathbf{M}$ & 8 & 8,1 \\
\hline Biofosfanatos & M05 & 3 & 2,0 \\
\hline $\begin{array}{l}\text { Medicamentos utilizados para o } \\
\text { tratamento da gota }\end{array}$ & M04 & 2 & 3,0 \\
\hline $\begin{array}{l}\text { Produtos anti-inflamatórios e } \\
\text { antirreumáticos }\end{array}$ & M01 & 2 & 2,0 \\
\hline Relaxantes musculares & M03 & 1 & 1,0 \\
\hline Sistema nervoso & $\mathbf{N}$ & 5 & 5,0 \\
\hline Analgésicos & N02 & 2 & 2,0 \\
\hline Psicoanaléptico & N06 & 2 & 2,0 \\
\hline Antiepiléticos & N03 & 1 & 1,0 \\
\hline Outros & B-G & 7 & 7,1 \\
\hline Total & & 99 & 100 \\
\hline
\end{tabular}

Frequências absolutas e relativas calculadas desconsiderando os medicamentos para os quais não foi possível uma classificação $(\mathrm{n}=21)$.
Dentre os usuários de medicamentos estudados, foi encontrada uma média de $2,1 \pm 1,5 \mathrm{RNM}$ s por indivíduo, podendo ser constatado que $81,5 \%$ deles correspondiam a problemas de efetividade e $13,2 \%$ por questóes de segurança não quantitativa do medicamento (Tabela 3 ).

Tabela 3: Resultados Clínicos Negativos Associados ao uso do Medicamento (RNM), segundo Consenso de Granada. Hipertensos da Farmácia e Fisioterapia da USCS - Município de São Caetano do Sul, SP, 2013.

\begin{tabular}{|c|c|c|}
\hline & Classificaçáo & $\begin{array}{l}\text { No de } \\
\text { RNM }\end{array}$ \\
\hline \multirow{2}{*}{ Necessidade } & $\begin{array}{l}\text { O paciente sofre um problema de } \\
\text { saúde em consequência de não receber } \\
\text { um medicamento de que necessita. }\end{array}$ & - \\
\hline & $\begin{array}{l}\text { O paciente sofre um problema de } \\
\text { saúde em consequência de receber um } \\
\text { medicamento de que náo necessita. }\end{array}$ & $2(5,3 \%)$ \\
\hline \multirow{2}{*}{ Efetividade } & $\begin{array}{l}\text { O paciente sofre um problema de } \\
\text { saúde em consequência de uma } \\
\text { inefetividade náo quantitativa do } \\
\text { medicamento. }\end{array}$ & $1(2,6 \%)$ \\
\hline & $\begin{array}{l}\text { O paciente sofre um problema } \\
\text { de saúde em consequência de } \\
\text { uma inefetividade quantitativa do } \\
\text { medicamento. }\end{array}$ & $30(78,9 \%)$ \\
\hline \multirow{2}{*}{ Segurança } & $\begin{array}{l}\text { O paciente sofre um problema de } \\
\text { saúde em consequência de uma } \\
\text { insegurança náo quantitativa de um } \\
\text { medicamento. }\end{array}$ & $5(13,2 \%)$ \\
\hline & $\begin{array}{l}\text { O paciente sofre um problema de } \\
\text { saúde em consequência de uma } \\
\text { insegurança quantitativa de um } \\
\text { medicamento. }\end{array}$ & - \\
\hline Total & & $38(100 \%)$ \\
\hline
\end{tabular}

No início do estudo, apenas $11,1 \%$ dos pacientes apresentavam pressão arterial controlada; no final do estudo, houve aumento proporcional dos hipertensos controlados $(72,2 \%)$, com redução do número de pacientes com hipertensão no estágio 2 e 3 (Tabela 4).

Tabela 4: Classificação da pressão arterial, de acordo com a VI Diretrizes Brasileiras de Hipertensão (2010), de hipertensos da Farmácia e Fisioterapia da USCS, no início e final da AF. Município de São Caetano do Sul - SP, 2013.

\begin{tabular}{lcccc}
$\begin{array}{l}\text { Classifi- } \\
\text { cação }\end{array}$ & $\begin{array}{c}\text { Pressáo } \\
\text { arterial } \\
\text { sistólica } \\
\text { (mmHg) }\end{array}$ & $\begin{array}{c}\text { Pressáo } \\
\text { arterial } \\
\text { diastólica } \\
(\mathbf{m m H g})\end{array}$ & $\begin{array}{c}\text { Número de } \\
\text { pacientes } \\
\text { início } \\
\text { da AF }\end{array}$ & $\begin{array}{c}\text { Número de } \\
\text { pacientes } \\
\text { fanal } \\
\text { da AF }\end{array}$ \\
$\begin{array}{l}\text { Pressão } \\
\text { normal }\end{array}$ & $<130$ & $<85$ & $2(11,1 \%)$ & $13(72,2 \%)$ \\
$\begin{array}{l}\text { Pressão } \\
\text { limítrofe }\end{array}$ & $130-139$ & $85-89$ & $4(22,2 \%)$ & $1(5,6 \%)$ \\
$\begin{array}{l}\text { Hipertensão } \\
\text { estágio 1 }\end{array}$ & $140-159$ & $90-99$ & $5(27,8 \%)$ & $4(22,2 \%)$ \\
$\begin{array}{l}\text { Hipertensão } \\
\text { estágio 2 }\end{array}$ & $160-179$ & $100-109$ & $4(22,2 \%)$ & - \\
$\begin{array}{l}\text { Hipertensão } \\
\text { estágio 3 }\end{array}$ & $\geq 180$ & $\geq 110$ & $3(16,7 \%)$ & - \\
\hline
\end{tabular}


A média das pressóes arteriais sistólicas do grupo estudado, ao final do acompanhamento, apresentou redução média de 23,8 mmHg. Ao comparar as médias inicial e final foi possível observar redução estatisticamente significativa $(\mathrm{p}<0,002)$. Quando comparada a pressão arterial sistólica entre os sexos, foi possível observar maior redução entre os indivíduos do sexo masculino $(39,7 \mathrm{mmHg})$. Entre o início e final do estudo, não foi encontrada, entre os indivíduos estudados, diferença estatisticamente significativa na pressão arterial diastólica (Tabela 5).

Tabela 5: Alterações na pressão arterial dos pacientes hipertensos da Farmácia e Fisioterapia da USCS, após intervenção da atenção farmacêutica. Município de São Caetano do Sul, SP, 2013.

\begin{tabular}{lccc}
$\begin{array}{l}\text { Pressão arterial } \\
(\mathbf{m m H g})\end{array}$ & $\begin{array}{c}\text { Início } \\
\text { Fca At. }\end{array}$ & Final *At. Fca & p \\
Ambos os sexos & & & \\
Sistólica & $151,6 \pm 25,8$ & $127,8 \pm 10,7$ & 0,002 \\
Diastólica & $77,4 \pm 21$ & $76,7 \pm 9,5$ & 0,88 \\
Masculino & & & \\
Sistólica & $151,7 \pm 17,2$ & $112 \pm 48,7$ & 0,002 \\
Diastólica & $82,7 \pm 17,9$ & $79,7 \pm 10,3$ & 0,73 \\
Feminino & & & \\
Sistólica & $151,5 \pm 29,8$ & $125,3 \pm 10,9$ & 0,002 \\
Diastólica & $74,8 \pm 22,7$ & $75,1 \pm 9,5$ & 0,96 \\
\hline
\end{tabular}

*At. Fca (Atenção Farmacêutica); resultado estatisticamente năo significativo $(\mathrm{p}>0,05)$.

\section{Discussão}

Atualmente muito se discute em relação ao aumento dos gastos em saúde impulsionado pelo custo dos medicamentos ${ }^{24}$, mas pouco se debate em definitivo quanto ao uso inadequado ${ }^{3}$ que conduz a agravos em saúde, consequentemente ao acréscimo desses gastos. Esse processo possivelmente é resultante do reflexo de uma sociedade que se encontra medicalizada e, polimedicada como a encontrada neste estudo, sendo necessário o aprofundamento de discussóes que possam trazer alternativas para solucionar esta equação.

$\mathrm{O}$ aumento da longevidade da população brasileira, principalmente em determinadas regióes, a exemplo do município de Sáo Caetano do Sul (19,1\% de idosos) ${ }^{25}$, deve ser trazido à luz desta discussáo, pois países europeus como a Espanha já apresentam esta realidade. Além de existir a situação de um novo paradigma demográfico, há também o predomínio de doenças crônicas não transmissíveis e degenerativas de aparição tardia, tais como o Alzheimer ${ }^{26}$, que em ambos os casos requerem terapias específicas. Esse fato por si só incrementa, em muito, os gastos em saúde, e este estudo buscou evidenciar tal evento demográfico, já que os pesquisados são pessoas idosas portadoras de doenças crônicas.

Fato importante a ser destacado, nesta pesquisa, é a falta de legibilidade das prescriçóes, evento também observado em outros estudos ${ }^{27,28}$, pois não se justifica o uso de receitas manualmente escritas em uma sociedade praticamente imersa na era da informática. Tratandose de uma população idosa, em muitos casos, hipertensa e polimedicada, como os indivíduos observados na FarmaUSCS, a não compreensão da descrição medicamentosa pode acarretar, como já se sabe, complicaçôes de saúde que elevam tanto os custos mensais da família, como também do sistema de saúde, despendendo ainda mais recursos materiais e humanos para solucionar um agravo que poderia ser evitado.

Entretanto, foi observado também que, possivelmente, parte da medicação encontrada nas prescriçōes não se encontrava repousada em uma evidência científica, pois na maioria das vezes não se levava em consideração possíveis interaçóes medicamentosas ${ }^{29}$ e reaçoos adversas que são inerentes do fármaco prescrito. Um dos exemplos é a existência da provável hiperuricemia secundária em indivíduos que utilizam tiazídicos, fato este observado nesta pesquisa. Outro exemplo é a utilização de inibidores da bomba de prótons, que na maioria das vezes são utilizados como "preventivo" em disfunçóes gástricas motivadas pela politerapia, houve prevalência de $33,3 \%$ nas prescriçôes estudadasNo momento da intervenção farmacêutica dispensada nesse grupo de pacientes, encontrou-se o conjunto desses fatores ora descritos, que conduziu a observação de que muito da inefetividade medicamentosa verificada antes da atenção farmacêutica exibia tais componentes. Durante o processo de pesquisa foi observado que a baixa adesão ao tratamento tinha como principal vinculação a ausência de informação sobre a hipertensão e o medicamento prescrito, o que, possivelmente, resultava no abandono farmacoterapêutico, apresentando como consequência o baixo controle da pressão arterial.

Além desses elementos, que possivelmente resultaram na baixa inefetividade quantitativa encontrada, estava a não adesão ao tratamento medicamentoso $(81,5 \%)$, diferentemente de outros estudos realizados anteriormente, em que foram observados resultados negativos associados ao medicamento por motivo dos participantes estarem utilizando um fármaco de que não necessitavam $(30,6 \%)$, como de efetividade $(33,2 \%)$, sendo esta possivelmente por falta de informação no momento da prescriçãoo ${ }^{17,18}$ e dispensação. Provavelmente essa diferença observada no que diz respeito à "necessidade" pode estar atrelada, no primeiro estudo, ao maior acesso aos medicamentos essenciais.

O resultante desse processo de identificação e resolução de problemas está na análise apresentada no 
momento anterior à intervenção farmacêutica, pois, no início deste estudo, $66,7 \%$ dos investigados foram classificados como hipertensos não controlados. Após a intervenção farmacêutica sobre os Problemas Relacionados aos Medicamentos encontrados, houve uma redução desse percentual para $22 \%$, quando comparado ao início da pesquisa.

Esses resultados positivos obtidos somente vêm corroborar para a importância da incorporação dessa prática no serviço público, que certamente resultaria em melhor qualidade de vida e economia para a sociedade. Muito se fala no custo elevado da saúde alavancado pelos medicamentos, mas pouco se tem discutido, no meio político-administrativo, sobre a relevância da incorporação desse cuidado farmacêutico como prática que pode levar à redução dos custos nesse setor.

Muitos já são os artigos que nos remetem à importância da intervenção farmacêutica no âmbito da saúde pública ${ }^{7,13}$, entretanto, parece que falta um pouco mais de ousadia desse serviço em aceitar a mudança do paradigma hegemônico até entáo para um paradigma definitivamente multi e interprofissional, em que o farmacêutico é também profissional importante na equipe de saúde. Esse profissional pode auxiliar na redução em parte desses gastos, como também proporcionar melhor qualidade de vida aos usuários de medicamentos mediante uma atenção farmacêutica qualificada. Afinal, essa não seria a finalidade do serviço de saúde pública?

Por meio deste estudo foi possível analisar que mesmo os pacientes possuindo acesso a seu tratamento medicamentoso, ainda assim em parte deles os níveis de pressão arterial náo se encontravam devidamente controlados.

\section{Conclusão}

Conclui-se por meio deste estudo que a atenção farmacêutica, realizada de maneira sistemática por profissional farmacêutico treinado para essa finalidade, pode contribuir efetivamente para o controle da pressão arterial dos usuários de medicamentos. E, muito provavelmente, uma intervenção farmacêutica ampliada em um município pode contribuir para a redução de hospitalizaçóes, possíveis sequelas e mortes prematuras motivadas pelo mau uso de medicamentos.

\section{Referências}

1. Agência Nacional de Vigilância Sanitária. Parcerias para diminuir o mau uso de medicamentos. Rev Saúde Pública. 2006;40(10):191-4.

2. Castro CGS, Teixeira CC. Interaçôes medicamentosas. In: Fuchas FD, Wannmacaher L, Ferreira MB. Farmacologia
Clínica - Fundamentos da terapêutica racional. $3^{\mathrm{a}}$ ed. Rio de Janeiro: Guanabara Koogan; 2006. p. 67-72.

3. Cipolle R, Strand LM, Morley P. El ejercicio de la atención farmacéutica. Madrid: McGraw Hill; 2000. 368p.

4. Hepller CD, Strand LM. Opportunities and responsabilities in pharmaceutical care. Am J. Hosp. Pharm. 1990;47:533-43.

5. Foppa AA, Bevilacqua G, Pinto LH, Blatt CR. Atenção farmacêutica no contexto da estratégia de saúde da família. Rev Bras Ciênc Farm. 2008;44(4):727-37.

6. Pereira LRL, Freitas O. A evolução da atenção farmacêutica e a perspectiva para o Brasil. Rev. Bras. Cienc. Farm. 2008;44(4).

7. Possamai FP, Dacoreggio MS. A habilidade de comunicação com o paciente no processo de atenção farmacêutica. Trab. Educ. Saúde. 2008;5(3):473-90.

8. Amariles M, Pedro GA, Newar TP, Victoria RG, Margarita GP, David PMJ. Método Dáder de seguimiento farmacoterapéutico y farmacovigilancia en instituciones de salud de Colombia. 2005;12(1/2):29-38.

9. Sabino W, Morell MGG. Estudo comparativo das desigualdades raciais ao morrer no Brasil. [trabalho na internet]. Trabalho apresentado no XVIII Encontro Nacional de Estudos Populacionais, ABEP; 19 a 23 de novembro de 2012 [acesso em 12 dez. 2014]; Águas de Lindoia, SP, Brasil. Disponível em: http://www.abep.nepo.unicamp.br/ xviii/anais/files/POSTER\%5B604\%5DABEP2012.pdf

10. Mansur AP, Souza MFM, Timerman A, Avakian SD, Aldrighi JM, Ramires AF. Trends in the risk of death from cardiovascular, cerebrovascular and ischemic diseases in thirteen States of Brazil from 1980 to 1998. Arq. Bras. Cardiol. 2006;87(5):641-8.

11. Rosa MLG, Giro C, Alves TO, Moura EC, Lacerda LS, SantAnna LP, et al. Análise da mortalidade e das internaçốes por doenças cardiovasculares em Niterói, entre 1998 e 2007. Arq. Bras. Cardiol. 2011;96(6):477-83.

12. Oliveira GMM, Klein CH, Nelson A. Mortalidade por doenças cardiovasculares em três estados do Brasil de 1980 a 2002. Rev Panam Salud Pública. 2006;19(2):85-93.

13. Provin MP, Campos AP, Nielson SEO, Amaral RG. Atenção Farmacêutica em Goiânia: inserção do farmacêutico na Estratégia Saúde da Família. Saúde Soc. 2010;19(3):717-23.

14. Souza TRCL, Silva AS, Leal LB, Santana DP. Método Dáder de seguimento terapêutico, terceira edição (2007): um estudo piloto. Rev. Cienc. Farm. Básica Apl. 2009;30(1):105-9.

15. Vinholes ER, Alano GM, Galato D. A percepção da comunidade sobre a atuação do Serviço de Atenção Farmacêutica em açóes de educação em saúde relacionadas à promoção do uso racional de medicamentos. Saúde Soc. 2009; 18(2):293-303.

16. Helena ETS, Nemes MIB, Eluf-Neto J. Avaliação da assistência a pessoas com hipertensão arterial em Unidades de Estratégia Saúde da Família. Saúde Soc. 2010;19(3):614-26. 
17. Vitor AF, Monteiro FPM, Morais HCC, Vasconcelos JDP, Lopes MVO, Araujo TL. Perfil das condiçóes de seguimento terapêutico em portadores de hipertensão arterial. Esc. Anna Nery. 2011;15(2):251-60.

18. Menezes TMO, Guimarães EP, Santos EMP, Nascimento MV, Araújo PD. Grupo educativo com dispensação de medicamentos: uma estratégia de adesão ao tratamento da hipertensão arterial e do diabetes mellitus. Rev. Baiana Saúde Pública. 2012;36(1):148-58.

19. Santos JC, Faria JM, Restini CBA. Potenciais interaçóes medicamentosas identificadas em prescriçóes a pacientes hipertensos. Rev. Soc. Bras. Clín. Méd. 2012;10(4):308-17.

20. Amarante LC, Shoji LS, Beijo LA, Lourenço EB, Marques LAM. A influência do acompanhamento farmacoterapêutico na adesão à terapia anti-hipertensiva e no grau de satisfação do paciente. Rev Ciênc Farm Básica Apl. 2010;31(3):209-15.

21. Sociedade Brasileira de Cardiologia. VI Diretrizes de Hipertensão. Arq Bras Cardiol. 2010;95(1):1-51.

22. Rosa MB, Neiva HM, Anacleto TA, Mendes DP, Freitas FO, Lage JB, et al. Legibilidade de prescrições médicas com medicamentos potencialmente perigosos em um hospital de Belo Horizonte. Rev. BRAFH. 2003;(2):22-8.

23. Faus Dáder MJ, Sabater Hernandéz D, Silva-Castro MM. Método Dáder. Guía de seguimiento farmacoterapéutico. $3^{a}$ ed. Granada: Universidad de Granada; 2007.

24. Kondro W. Drug spending hits $\$ 24.8$ billion. CMAJ. 2006;175(1):22.

25. Instituto Brasileiro de Geografia e Estatística. Censo Demográfico 2010. Rio de Janeiro: IBGE; 2011.

26. Regidor E, Fisac JLG, Calle ME, Otero A. Patrón de mortalidad en España. Med Clin. Barcelona. 2002;118(1):13-5.

27. Araújo PTB, Uchôa SAC. Avaliação da qualidade da prescrição de medicamentos de um hospital de ensino. Ciênc. Saúde Coletiva. 2011;16(Suppl 1):1107-14.

28. Silvério MS, Leite ICG. Qualidade das prescriçōes em município de Minas Gerais: uma abordagem farmacoepidemiológica. Rev. Assoc. Med. Bras. 2010;56(6):675-80.

29. Moreno AH, Nogueira EP, Perez MPMS, Lima LRO. Atenção farmacêutica na prevenção de interaçóes medicamentosas em hipertensos. Rev. Ins Ciên Saúde. 2007;25(4):373-7.

\section{Como citar este artigo:}

Silva LGA, Aragão CCV, Sabino W. Pressão arterial e atenção farmacêutica: o cuidado faz a diferença. Rev. Aten. Saúde. 2016;14(47): 12-18. 